Original Paper http://ajol.info/index.php/ijbcs http://indexmedicus.afro.who.int

\title{
Essai préliminaire de production d'œufs des poules pondeuses (ISA Warren) enrichis en acides gras polyinsaturés oméga 3 avec les graines de Euphorbia heterophylla $\mathrm{L}$.
}

\author{
N'Goran David Vincent KOUAKOU ${ }^{1 *}$, Gnantro Cheick TRAORE ${ }^{1}$, \\ Cho Euphrasie Monique ANGBO-KOUAKOU ${ }^{2}$, Kouadio Bertin KOUAME ${ }^{1}$, \\ Amissa Augstin ADIMA ${ }^{3}$, Nogbou Emmanuel ASSIDJO ${ }^{3}$, Jean-François GRONGNET ${ }^{4}$ \\ et Maryline KOUBA ${ }^{4}$ \\ ${ }^{1}$ Département de Formation et de Recherche Agriculture et Ressources Animales, Institut National \\ Polytechnique Félix Houphouët-Boigny, B.P. 1313 Yamoussoukro, Côte d'Ivoire. \\ ${ }^{2}$ Département de Formation et de Recherche Gestion Commerce Economie Appliquée, Institut National \\ Polytechnique Félix Houphouët-Boigny, B.P. 1313 Yamoussoukro, Côte d'Ivoire. \\ ${ }^{3}$ Département de Formation et de Recherche Génie chimique Agro-alimentaire, Institut National Polytechnique \\ Félix Houphouët-Boigny,. B.P. 1313 Yamoussoukro, Côte d'Ivoire. \\ ${ }^{4}$ Unité Mixte Recherche (UMR) PEGASE INRA-Agrocampus Ouest, 65 rue de Saint-Brieuc, 35042 Rennes \\ cedex. France. \\ *Auteur correspondant ; E-mail: kwayki@yahoo.fr ; B.P. 1313 Yamoussoukro (RCI) ; Tél: +22508 393363 ; \\ fax: +22530640406 ; cel: +22502033581 ; dom: +22530645120
}

\section{RESUME}

En Afrique, la notion de qualité nutritionnelle des œufs de poules pondeuses semble être abstraite. Afin d'y remédier, cette étude vise à déterminer l'impact d'un régime supplémenté en graines de Euphorbia heterophylla sur la qualité nutritionnelle d'œufs de poules pondeuses. A cet effet, vingt-quatre poules (ISA Warren) de 1,4 kg ont reçu durant 28 jours soit un régime contrôle (RC) (100\% d'aliment commercial), soit un régime expérimental (R15) (80\% de RC, 5\% de complément minéral vitaminé et $15 \%$ de graines de Euphorbia heterophylla). Les dosages effectués ont montré que le régime $\mathrm{R} 15$ a conduit à une baisse significative des proportions d'acides gras saturés, monoinsaturés, polyinsaturés n-6 et a induit à une augmentation significative de la proportion en acides gras polyinsaturés n-3. Les proportions des acides gras C18:3 n-3, C20:5 n-3, C22:5 n-3 et C22:5 n-3 des jaunes des œufs issus de R15 ont été multipliées respectivement par 31, 10, 4 et 3. Une réduction significative de $18 \%$ du taux de cholestérol total a été observée avec le régime expérimental $\mathrm{R} 15$. La consommation de ce type d'œuf permettrait d'améliorer la prévention des maladies cardiovasculaires, principales causes de mortalité dans les pays africains à revenu faible ou intermédiaire.

(C) 2015 International Formulae Group. All rights reserved.

Mots clés : Eufs, acides gras, cholestérol, nutrition, santé. 


\title{
Preliminary assay of production of layers' eggs (ISA Warren) enriched in polyinsaturated fatty acids omega 3 using Euphorbia heterophylla seeds
}

\begin{abstract}
In Africa, notion of nutritional quality of layers' eggs seems to be abstract. To remedy it, this study aims to determining the effect of a diet supplemented with seeds of Euphorbia heterophylla on the nutritional quality of layers' eggs. For this purpose, twenty-four hens (ISA Warren) 1,4 kg for 28 days received either a control diet (RC) (100\% food marketed) or an experimental diet (R15) (80\% RC, 5\% vitamin and mineral supplement $15 \%$ seeds of Euphorbia heterophylla). The analysis showed that R15 diet has significantly led to a decline in proportions saturated fatty acids, monounsaturated fatty acids, omega- 6 PUFA and induced an significantly increase of the proportion of n-3 PUFA. The proportions of fatty acids C18:3 n-3, C20:5 n-3, C22:5 n-3 and C22:5 n-3 of yellow eggs from R15 have increased respectively by 31, 10, 4 and 3 . A significantly reduction of $18 \%$ to cholesterol the egg yolk was observed with the experimental diet R15. The consumption of this type of egg will allow improve prevention of cardiovascular disease, leading cause of death in low- and middle-income.
\end{abstract}

(c) 2015 International Formulae Group. All rights reserved.

Keywords: Eggs, fatty acid, cholesterol, nutrition, health.

\section{INTRODUCTION}

Les maladies cardiovasculaires sont la première cause de mortalité $(31 \%)$ dans le monde (OMS, 2014). Elles tuent deux fois plus que le VIH/SIDA, le paludisme et la tuberculose réunis dans l'ensemble des pays en voie de développement (Abegunde et al., 2007). En 2014, approximativement 17,5 millions de personnes sont décédées de maladies cardiovasculaires dans le monde dont plus des trois quarts dans les pays à revenu faible ou intermédiaire (OMS, 2014). Un des facteurs de risque est l'élévation du taux de cholestérol liée à une consommation excessive de graisses saturées et de cholestérol (German et Dillard, 2004). Ce sont les produits animaux (cervelle, œufs, abats, viande, foie gras, beurre,...) qui sont les plus riches en cholestérol car seuls les animaux peuvent le synthétiser (ANSES, 2011). Parmi les produits animaux, l'œuf avec sa teneur en cholestérol de $5 \mathrm{mg} / \mathrm{g}$ d'œuf contient 8 fois plus de cholestérol que la viande de porc (Bourre, 2005). Un moyen de diminuer la teneur en cholestérol de l'œuf est de supplémenter le régime alimentaire de la poule en germes de maïs (Kovacs et al., 2000) ou en sources d'acides gras polyinsaturés oméga 3 (AGPI n-3) comme la graine de lin (Sari et al., 2002).

Les acides gras polyinsaturés oméga 3 sont essentiels pour l'homme et ont un rôle reconnu dans la prévention des maladies coronariennes (ANSES, 2011). Un moyen d'augmenter la consommation d'acides gras polyinsaturés oméga 3 est d'enrichir l'œuf en ces acides gras en supplémentant le régime de la poule comme l'ont fait de nombreux auteurs (revue de (Ahmad et al., 2012)). En Côte d'Ivoire, il a été récemment démontré que Euphorbia heterophylla, une plante adventice, distribuée au cochon d'Inde (Cavia porcellus L.) en complément de Panicum maximum conduit à un enrichissement en acides gras polyinsaturé (AGPI) n-3 de la carcasse (Kouakou et al., 2013). Afin de tester la possibilité d'enrichissement en acides gras polyinsaturés (AGPI) n-3 des œufs de poules pondeuses à partir des graines de Euphorbia heterophylla, cette étude a été menée. Elle a pour objectif de déterminer l'effet d'un régime supplémenté en graines de Euphorbia heterophylla sur la composition en acides gras ainsi que la teneur en cholestérol des oufs de poules pondeuses. 


\section{MATERIEL ET METHODES}

L'étude a été conduite à la ferme expérimentale du Département Agriculture et Ressources Animales de l'Institut National Polytechnique Félix Houphouët Boigny (INPHB) $\left(6,5^{\circ} \mathrm{N} ; 5,2^{\circ} \mathrm{O}\right)$. La température et l'humidité relative durant l'essai ont varié respectivement de 20 à $30{ }^{\circ} \mathrm{C}$ et de 80 et $85 \%$. La pluviométrie moyenne mensuelle est de $1100 \mathrm{~mm}$.

\section{Animaux et régimes à tester}

Deux lots de 12 poules ISA Warren de 8 mois provenant de la ferme expérimentale de l'Institut National Polytechnique Félix Houphouët-Boigny (INP-HB) en Côte d'Ivoire ont été soumis à deux régimes, un régime commercial contrôle (RC) $(100 \%$ IVOGRAIN-ponte 20 SIPRA, Côte d'Ivoire) ou un régime expérimental composé du régime commercial contrôle (RC) $(80 \%)$ supplémenté par $5 \%$ de complément minéral vitaminé et $15 \%$ de graines de Euphorbia heterophylla (R15) (Tableaux 1 et 2).

\section{Mode d'élevage}

Les poules ont été élevées en cages en bois de $3,375 \mathrm{~m}^{3}(1,5 \times 1,5 \times 1,5 \mathrm{~m})$ sur litière, à raison de quatre poules par cage, et trois cages par régime. Les mesures de prophylaxie sanitaire ont été respectées afin de prévenir l'apparition d'éventuelles pathologies. Les poules étaient de poids moyen équivalent dans les deux lots (environ $1,44 \mathrm{~kg}$ ) et ont reçu $120 \mathrm{~g}$ d'aliment et de l'eau à volonté. L'essai a duré 28 jours. A la fin de l'expérimentation, tous les animaux ont été abattus.

\section{Prélèvements et analyses des échantillons}

Les œufs des deux dernières semaines ont été collectés par régime et par cage. A la fin de l'essai, six œufs par cage ont été choisis de manière aléatoire, puis cassés. La coquille, l'albumen et le vitellus ont été séparés. Les six jaunes d'œufs par cage ont été mélangés puis congelés avant analyses. Les lipides des jaunes d'œufs ont été extraits selon la technique de Delsal (1944). Ceux des aliments expérimentaux l'ont été par la méthode de Folch et al. (1957). Les profils en acides gras des aliments expérimentaux et des jaunes d'œufs ont été déterminés selon la technique de Morrison et Smith (1964) par chromatographie en phase gazeuse. La teneur en cholestérol des jaunes d'œufs a été mesurée à l'aide d'un kit enzymatique (Cat. No., 11491458, Roche cholesterol assay).

Les teneurs en matières minérales (cendres brutes) des différents régimes expérimentaux ont été déterminées suivant les méthodes de la norme de l'Association Officielle des Chimistes Analytiques (AOAC, 2006) et celles des protéines brutes ont été obtenues par la méthode de Kjeldahl ( $\mathrm{N}$ x 6,25). Quant à la teneur cellulose brute, elle a été déterminée suivant la norme AOAC (2006) fondée sur la méthode de Weende. La détermination de la teneur en Calcium (Ca) et Phosphore (P) dans les régimes expérimentaux a été réalisée par la méthode spectrophotométrique d'absorption (AOAC, 2006).

\section{Analyses statistiques}

Les différences entre les deux lots ont été testées par le test-t de Student (STATA, 2008). L'effet fixe était le régime. Le seuil de signification était de 5\%.

\section{RESULTATS ET DISCUSSION}

Composition chimique et profils en acide gras des aliments expérimentaux

La composition chimique de ces deux régimes expérimentaux est présentée dans le Tableau 1. Les régimes étaient iso-protéiques et avaient la même énergie métabolisable. Les profils d'acides gras des régimes étaient très différents. Les proportions en acides gras saturés (AGS) et acides gras mono-insaturés (AGMI) étaient plus faibles dans le régime $\mathrm{R} 15$. Le régime témoin avait environ deux fois plus d'acide oléique (C18:1 n-9) et une teneur supérieure en acide linoléique (C18:2 n-6) que le régime R15. Par contre, la teneur en acides gras polyinsaturés (AGPI) dans le 
régime R15 était plus élevée, en particulier celle en acide linolénique (C18:3 n-3), seize fois plus importante que dans le régime témoin.

\section{Teneur en lipides totaux et en cholestérol total des jaunes d'œufs}

Les teneurs en lipides totaux des jaunes d'œufs n'ont pas été affectées par les régimes testés $(\mathrm{P}>0,05)$. Des résultats identiques ont été également observés avec des régimes supplémentés en graines de lin (Augustyn et al., 2006). Le régime $\mathrm{R} 15$ a induit une réduction de $18 \%$ de la teneur en cholestérol total $(\mathrm{P}<0,05)$ (Tableau 2). Cette baisse de la teneur en cholestérol des jaunes d'œufs a été constatée par Ayerza et Coates (2000) en supplémentant le régime de poules avec du chia (Silva hispanica L.). Ces résultats diffèrent de ceux obtenus par certains auteurs qui n'ont constaté aucune différence significative de la teneur en cholestérol des jaunes d'œufs (Ansari et al., 2006; Yalcyn et al., 2007).

\section{Profil en acide gras des jaunes d'œufs}

Le régime $\mathrm{R} 15$ a conduit à une baisse des proportions d'acides gras saturés (AGS), des acides gras monoinsaturés (AGMI) et des acides gras polyinsaturés oméga 6 (AGPI oméga 6) $(\mathrm{P}<0,05)$. En effet, l'augmentation de la teneur en acides gras polyinsaturés oméga 3 (AGPI n-3) a provoqué une baisse des proportions des AGS, AGMI et AGPI n-6, ce qui est conforme aux résultats obtenus par Ayerza et Coates (2000) avec le chia (Silva hispanica L.) et à plusieurs études utilisant une supplémentation par du lin (revue de (Ahmad et al., 2012)).

Le régime R15 également induit une teneur en acide gras polyinsaturés oméga 3 (AGPI n-3) environ neuf fois $(8,95)$ plus importante que dans le régime contrôle (RC) $(\mathrm{P}<0,05)$. Les proportions en acide linolénique (C18:3 n-3, ALA), en acide eicosapentaénoïque (C20:5 n-3, EPA), en acide clupanodonique C22:5 n-3 (DPA) et en acide docosahexaénoïque (C22:6 n-3, DHA) des jaunes des œufs issus du régime R15 ont été multipliées respectivement par 31, 10, 4 et 3. L'augmentation de la teneur en acide gras polyinsaturés oméga 3 (AGPI n-3), en acide linolénique (C18:3 n-3, ALA) et en acide docosahexaénoïque (C22:6 n-3, DHA) du jaune d'œuf de poule recevant un régime riche en AGPI n-3 a déjà été décrite (Bean et Leeson, 2003; Ayerza et Coates, 2000). Cependant, contrairement à ces auteurs, la présente étude montre une augmentation significative de la teneur en acide eicosapentaénoïque (C20:5 n-3, EPA). Selon Ayerza et Coates (2000), l'absence de cet acide gras pourrait être attribuée à la limite de seuil de détection chromatographique plutôt qu'à son absence réelle et cela en raison des proportions relatives assez faibles de cet acide gras. En effet, l'acide eicosapentaénoïque (C20:5 n-3, EPA) représente qu'une étape de la conversion de l'acide linolénique (C18:3 n3, ALA) en acide docosahexaénoïque (C22:6 n-3, DHA) (ANSES, 2011).

Le régime $\mathrm{R} 15$ a conduit à une forte diminution du rapport des acides gras C18:2 n-6/C18:3 n-3 (41,7 pour le régime témoin contre 1,2 pour le régime $\mathrm{R} 15) \quad(\mathrm{P}<0,05)$ (Tableau 2). Le rapport des acides gras $\mathrm{C} 18: 2$ n-6/C18:3 n-3 du jaune d'œuf induit par le régime R15 est inférieur à ceux de Ayerza et Coates (2000) utilisant comme source d'enrichissement des graines de chia (Salvia hispanica L.) à un taux d'incorporation de $21 \%$ après 30 jours de distribution. Ce résultat confirme l'intérêt nutritionnel de cette supplémentation en graines de Euphorbia heterophylla de l'alimentation des poules pondeuses et est en total accord avec les recommandations des nutritionnistes qui souhaitent un ratio C18:2 n-6/C18:3n-3 dans le régime alimentaire de l'homme inférieur ou égale à 5 (ANSES, 2011). Aussi, la consommation de ce type d'œuf permettraitelle d'améliorer la prévention des maladies cardiovasculaires, principales causes de mortalité dans les pays africains à revenu faible ou intermédiaire. 
Tableau 1: Ingrédients, compositions chimiques et profils en acides gras des différents régimes des poules pondeuses (régime contrôle (RC) ou supplémenté par 15\% de graines d'Euphorbia heterophylla (R15)).

\begin{tabular}{|c|c|c|}
\hline \multirow{2}{*}{ Ingrédients (\%) } & \multicolumn{2}{|c|}{ Régimes expérimentaux } \\
\hline & RC & R15 \\
\hline Ivograin Ponte 20 & 100 & 80,00 \\
\hline Euphorbia heterophylla (graine) & - & 15,00 \\
\hline Phosphate bicalcique & - & 4,8 \\
\hline Lysine & - & 0,2 \\
\hline \multicolumn{3}{|l|}{ Compositions chimiques (\%) } \\
\hline Protéine brute & 18,93 & 18,93 \\
\hline Matière grasse & 1,89 & 6,38 \\
\hline Matière minérale & 12,83 & 15,72 \\
\hline Cellulose brute & 1,58 & 2,89 \\
\hline Calcium & 3,58 & 4,64 \\
\hline Phosphore & 1,79 & 2,76 \\
\hline Calcium/Phosphore* & 2,01 & 1,68 \\
\hline Energie métabolisable $(\mathrm{Mj} / \mathrm{kg} \mathrm{MS})^{*}$ & 14,17 & 14,17 \\
\hline \multicolumn{3}{|c|}{ Profils en acides gras (\% des acides gras totaux) } \\
\hline$\Sigma$ AGS & 23,97 & 18,15 \\
\hline C14:0 & 0,25 & 0,12 \\
\hline $\mathrm{C} 15: 0$ & 0,08 & 0,04 \\
\hline C16:0 & 18,51 & 11,99 \\
\hline C18:0 & 4,59 & 5,71 \\
\hline $\mathrm{C} 20: 0$ & 0,54 & 0,29 \\
\hline$\Sigma$ AGMI & 31,53 & 16,33 \\
\hline C16:1 n-9 & 0,15 & 0,04 \\
\hline C16:1 n-7 & 0,47 & 0,19 \\
\hline C18:1 n-9 & 29,43 & 14,93 \\
\hline C18:1 n-7 & 1,12 & 0,96 \\
\hline C20:1 n-9 & 0,36 & 0,21 \\
\hline$\Sigma$ AGPI & 44,51 & 65,52 \\
\hline C18:2 n-6 & 42,05 & 25,38 \\
\hline C18:3 n-3 & 2,46 & 40,14 \\
\hline $18: 2 n-6 / 18: 3 n-3$ & 17,0934959 & 0,632287 \\
\hline
\end{tabular}


Tableau 2: Teneur en lipides (en \%) et cholestérol total ( $\mathrm{mg} / \mathrm{g}$ ) et composition en acides gras (\% des acides gras totaux) du vitellus des œufs de poules recevant un régime contrôle (RC) ou supplémenté par 15\% de graines d'Euphorbia heterophylla (R15).

\begin{tabular}{|c|c|c|c|}
\hline \multirow{2}{*}{ Profils } & \multicolumn{2}{|c|}{ Régimes expérimentaux } & \multirow{2}{*}{ Effet du régime } \\
\hline & $\mathbf{R C}$ & $\mathbf{R} 15$ & \\
\hline Lipides totaux & 30,5 & 28,2 & NS \\
\hline Cholestérol total & 24,3 & 20,0 & $*$ \\
\hline \multicolumn{4}{|l|}{ Acides gras } \\
\hline$\Sigma \mathrm{AGS}$ & 35,8 & 34,8 & $*$ \\
\hline$\Sigma \mathrm{AGMI}$ & 48,8 & 41,5 & $* * *$ \\
\hline$\Sigma$ AGPI n-3 & 1,05 & 11,4 & $* * *$ \\
\hline C18:3 n-3 & 0,29 & 9,06 & $* * *$ \\
\hline $\mathrm{C} 20: 5 \mathrm{n}-3$ & 0,01 & 0,10 & $* * *$ \\
\hline $\mathrm{C} 22: 5 \mathrm{n}-3$ & 0,08 & 0,31 & $* * *$ \\
\hline $\mathrm{C} 22: 6 \mathrm{n}-3$ & 0,58 & 1,77 & $* *$ \\
\hline$\Sigma$ AGPI n-6 & 14,0 & 12,2 & $* *$ \\
\hline C18:2 n-6 & 12,1 & 10,9 & $*$ \\
\hline C20:4 n-6 & 1,40 & 0,89 & $*$ \\
\hline $\mathrm{C} 22: 4 \mathrm{n}-6$ & 0,14 & 0,07 & $* *$ \\
\hline$\Sigma \mathrm{AGPI} / \Sigma \mathrm{AGS}$ & 0,42 & 0,68 & $* * *$ \\
\hline $18: 2 n-6 / 18: 3 n-3$ & 41,7 & 1,20 & $* * *$ \\
\hline $\begin{array}{l}\Sigma \text { AGS: somme de } \\
\Sigma \text { AGMI: somme } \\
\Sigma \text { AGPI: somme d } \\
\Sigma \text { AGPI n-3 : somr } \\
\Sigma \text { AGPI n-6 : somr } \\
\text { NS: non significati }\end{array}$ & $\begin{array}{l}\text { s (C14:0- } \\
\text { oinsaturé } \\
\text { nsaturés } \\
\text { olyinsat } \\
\text { olyinsat } \\
0.05 ; *\end{array}$ & $\begin{array}{l}18: 0+\mathrm{C} \\
-\mathrm{C} 18: 1+ \\
\text { FA), } \\
\text { C18: } 4+C \\
\mathrm{C} 18: 3+\mathrm{C} \\
<0.001\end{array}$ & $\begin{array}{l}20: 5+C 22: 5+C 22: 6) \\
20: 4+C 22: 4)\end{array}$ \\
\hline
\end{tabular}

\section{Conclusion}

La supplémentation des graines de Euphorbia heterophylla dans le régime de poules a des effets importants sur la qualité nutritionnelle des œufs (augmentation de la teneur en acide gras polyinsaturés oméga 3 (AGPI n-3), baisse de la teneur en cholestérol). Cependant, d'autres études devront être réalisées afin de confirmer les présents résultats sur un plus grand nombre de volailles.

\section{CONFLITS D'INTERETS}

Les auteurs déclarent qu'ils n'ont aucun conflit d'intérêt.

\section{CONTRIBUTIONS DES AUTEURS}

Les auteurs N'DVK, GCT, CEMA-K, KBK, AAA, NEA et J-FG ont contribué de manière significative à la conception et à la mise en place du plan de travail et à la collecte des données. N'DVK, GCT, CEMA-K et MK ont contribué de manière significative à la collecte des données, à l'analyse et interprétation des résultats. Tous les auteurs suscités ont participé à l'organisation des idées, à la révision du contenu intellectuel du document et sont à mesure d'en défendre individuellement le contenu. 


\section{REMERCIEMENTS}

Les coauteurs tiennent à remercier toutes les personnes physiques ou morales qui ont contribué de près ou de loin à l'obtention des présents résultats.

\section{RÉFÉRENCES}

Abegunde DO, Mathers CD, Adam T, Ortegon M., Strong K. 2007. The burden and costs of chronic diseases in low-income and middle-income countries. Lancet, 370: 1929-1938. DOI : http://dx.doi.org/10.1016/S01406736(07)61696-1

Ahmad S, Haq AU, Yousaf M, Sabri MA, Kamran Z. 2012. Response of laying hens to omega-3 fatty acids for performance and egg quality. Avian Biology Research, 5(1): 1-10. DOI: $10.3184 / 175815512 X 132915061$ 28070

Ansari R, Azarbayejani A, Ansari S, Asgari S, Gheisari A. 2006. Production of egg enriched with omega- 3 fatty acids in laying hens. Arya Journal, 1(4): 242246. http://www.sid.ir/en/VEWSSID/ J_pdf/106120060402.pdf

ANSES (Agence nationale de sécurité sanitaire de l'alimentation, de l'environnement et du travail) 2011. Actualisation des apports nutritionnels conseillés pour les acides gras. Rapport d'expertise collective. Paris.

AOAC (Association Officielle des Chimistes Analytiques). 2006. Official Methods of Analysis $\left(18^{\text {th }}\right.$ edn). Association of Official Analytical Chemists: Arlington, USA.

Ayerza R, Coates W. 2000. Dietary Levels of Chia: influence on yolk cholesterol, lipid content and fatty acid composition for two strains of hens. Poultry Science, 79: 724-739.
Augustyn R, Barteczko J, Smulikowska S. 2006. The effect of feeding regular or low $\alpha$-linolenic acid linseed on laying performance and total cholesterol content in eggs. Journal of Animal Feed Sciences, 15: 103-106.

Bean ID, Leeson S. 2003. Long-term effect of feeding linseed on performance and egg fatty acid composition of brown and white hens. Poultry Science, 82: 388-394. DOI : $10.1093 / \mathrm{ps} / 82.3 .388$

Bourre J-M, 2005. Enrichissement de l'alimentation des animaux avec les acides gras oméga-3 : impact sur la valeur nutritionnelle de leurs produits pour l'homme. Médecine science : M/S, 21(8-9): 773-779.

Delsal L. 1944. A new procedure for extraction of serum lipids with methylal. Application to microdetermination of total cholesterol, phosphoaminolipids and proteins. Bulletin de la Société de Chimie et de Biologie, 26: 99-105.

Folch J, Lees M, Stanley GHS. 1957. A simple method for the isolation and purification of total lipids from animal tissues. Journal of Biological Chemestry, 226: 497-509.

German JB, Dillard CJ. 2004. Saturated fats: what dietary intake? American Journal of Clinical Nutrition, 80(3): 550-559.

Kouakou NDV, Grongnet J-F, Assidjo EN, Thys E, Marnet P-G, Catheline D, Legrand P, Kouba M. 2013. Effect of a supplementation of Euphorbia heterophylla on nutritional meat quality of Guinea pig (Cavia porcellus L.). Meat Science, 93(4): 821-826. DOI : 10.1016/j.meatsci.2012.11.036

Kovacs G, Schmidt J, Husveth F, Dublecz K, Wagner L, Farkas-Zelz E. 2000. Effect of feed composition on cholesterol 
content of the table eggs. Acta Alimentaria, 29: 25-41.

Morrison WR, Smith LM. 1964. Preparation of fatty acid methyl esters and dimethylacetals from lipids with boron fluoride-methanol. Lipids Research, 5: 600-608.

Organisation Mondiale de la Santé (OMS) 2014. Global status report on noncommunicable diseases.

Sari M, Aksit M, Özdogan M, Basmacioglu H. 2002. Effects of addition of flaxseed to diets of laying hens on some production characteristics, levels of yolk and serum cholesterol, and fatty acid composition of yolk. Archiv fur Geflügelkunde, 66(2): 75-79.

STATA. 2008. Stata/IC 10.0 for windows, StataCorp LP. Lakeway Drive College Station, TX, USA.

Yalcyn H, Unal MK, Basmacyoolu H. 2007. The fatty acid and cholesterol composition of enriched egg yolk lipids obtained by modifying hens' diets with fish oil and linseed. Journal of Medicinal Food, 58(4): 372-378. 